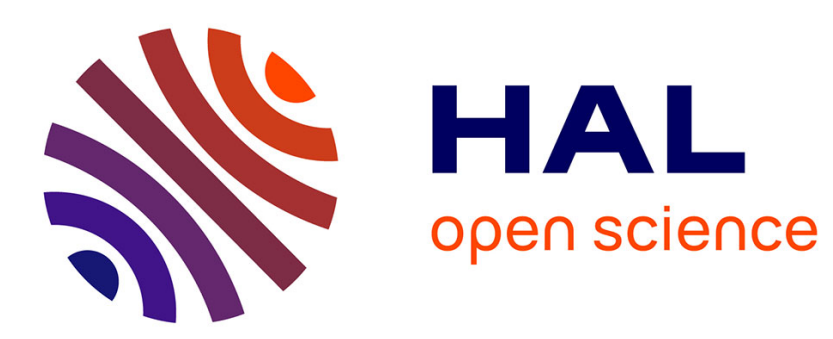

\title{
Advanced Synchronization of Audio or Symbolic Musical Patterns: An Algebraic Approach
}

Florent Berthaut, David Janin, Benjamin Martin

\section{To cite this version:}

Florent Berthaut, David Janin, Benjamin Martin. Advanced Synchronization of Audio or Symbolic Musical Patterns: An Algebraic Approach. International Journal of Semantic Computing, 2012, 6 (4), pp.409-427. 10.1142/S1793351X12400132 . hal-00794196

\section{HAL Id: hal-00794196 https://hal.science/hal-00794196}

Submitted on 25 Feb 2013

HAL is a multi-disciplinary open access archive for the deposit and dissemination of scientific research documents, whether they are published or not. The documents may come from teaching and research institutions in France or abroad, or from public or private research centers.
L'archive ouverte pluridisciplinaire $\mathbf{H A L}$, est destinée au dépôt et à la diffusion de documents scientifiques de niveau recherche, publiés ou non, émanant des établissements d'enseignement et de recherche français ou étrangers, des laboratoires publics ou privés. 
LaBRI, CNRS UMR 5800

Laboratoire Bordelais de Recherche en Informatique

Rapport de recherche RR-1461-12 (revised october 2012)

\section{Advanced Synchronization of Audio or Symbolic Musical Patterns: An Algebraic Approach}

February 25, 2013

Florent Berthaut, David Janin and Benjamin Martin

LaBRI, Université de Bordeaux, 351 , cours de la libération, F-33405 Talence, France

\{berthaut|janin|martin\}@labri.fr 


\section{Contents}

1 Introduction $\quad 3$

2 The advanced synchronization model 4

2.1 Basic pattern model ... . . . . . . . . . . . . . . 4

2.2 Towards an extended model . . . . . . . . . . . . . . 5

2.3 Realization and synchronization windows . . . . . . . . . 6

2.4 Sequential composition . . . . . . . . . . . . . . . 7

2.5 Resynchronization operator ................. 9

3 Derived and additional operators 10

3.1 Context patterns and left and right shifts . . . . . . . . . 10

3.2 Fork and join . . . . . . . . . . . . . . . . . . . . . 11

3.3 Expansion/contraction operator . . . . . . . . . . . . 11

3.4 Parallel and extended parallel products . . . . . . . . . . . 12

4 Application to audio patterns: advanced recomposition 13

4.1 Audio advanced synchronization . . . . . . . . . . . . . 13

4.2 Application to audio recomposition . . . . . . . . . . . . 14

5 Application to musical performance: Advanced Live-Looping 17

5.1 Model of control live-looping . . . . . . . . . . . . . . . . . 17

5.2 Recalage: an advanced control live-looper . . . . . . . . . . 18

6 Conclusion $\quad 20$ 


\title{
Advanced Synchronization of Audio or Symbolic Musical Patterns: An Algebraic Approach
}

\author{
Florent BERTHAUT, David JANIN and Benjamin MARTIN \\ LaBRI, Université de Bordeaux, \\ 351, cours de la libération, \\ F-33405 Talence, France \\ \{berthaut | janin|martin\}@labri.fr
}

February 25, 2013

\begin{abstract}
We define a generic model for finite audio or symbolic musical patterns that structurally encodes a rich and abstract synchronization mechanism. This is achieved by distinguishing for each pattern a realization window, describing what the pattern is, from a synchronization window, describing how the pattern can be used. The sequential composition of patterns is defined and studied. An algebra of musical patterns is introduced in a mathematically well-founded approach. We propose several high level operators that can be used either in audio processing or in musical analysis and composition. Practical uses and experiments conducted in both fields are described.
\end{abstract}

\section{Introduction}

The last decades have seen the development of various software programs for Computer Assisted Music either used on stage for live performances or integral to multimedia applications for rich interactive audio supports. These software solutions range from low level sound synthesis and control tools such as Faust [10] or Max/MSP [5], to high level composition assistants such as Elody [18] or OpenMusic [1] to name but a few.

In some rough dichotomy, low level (audio) software applications manage the nature of sounds: at any time quantum, they provide its value. On the opposite side, high level (music) applications manage the structure of music: it is defined as some combination of notes, motives, movements, etc., each with a specific duration, dynamics, usage, etc. As there is an increasing need of mixed usage of both software types, there is also an increasing need of structuring sounds in such a way that high-level compositions of musical patterns can be translated into 
low-level partial superimpositions of audio patterns. This becomes especially crucial when interactive musical pieces are to be defined and performed $[6,12]$.

In this paper, we consider the problems of synchronizing musical patterns, in either the audio or symbolic case. By discriminating between the definition of the pattern (what music is to be played) and its usage (when music is to be played), we define an advanced synchronization mechanism. It essentially consists in distinguishing, for every pattern, a realization window from a synchronization window. Sequentially combining two audio or symbolic patterns then amounts to sequentially combining their synchronization windows. As a result, realization windows may overlap. The handling of overlapping patterns, which is critical for a practical use of advanced synchronization, is defined according to the application.

In other words, we introduce a kind of musical pattern algebra, deeply wellfounded from a mathematical point of view. Once presented this algebra (Sec-

tions 2 and 3), we illustrate its relevance for musical applications by means of two case studies: automatic audio recomposition (Section 4) and live looping performance (Section 5).

\section{The advanced synchronization model}

In this section, we define our generic model of musical patterns with advanced synchronization information.

\subsection{Basic pattern model}

In both signal processing or computational music, an audio or symbolic pattern can be abstracted as the mapping

$$
S:[0, d] \rightarrow A \cup\{\perp\},
$$

such that $[0, d] \subseteq \mathbb{R}$ is the time interval on which $S$ is defined, $A$ is the (finite) set of values this pattern may take at a given time and $\perp$ corresponds to an undefined value.

For convenience, we represent $S$ on a relative time scale, hence starting at date 0 and lasting $d \in \mathbb{R}$. When modeling digital audio patterns, $A$ is the set of possible sample values, depending on the audio quantization used. When modeling symbolic music, $A$ is the set of all sets of control events that may be played at the same time.

One may remark that in both audio and symbolic cases, the definition domain $\operatorname{dom}(S)$ is, a priori, a finite subset of $[0, d]$ either defined by the sample rate of the audio pattern, or defined by the event dates of the control pattern. In other words, a pattern $S$ is a partial function from $[0, d]$ to $A$. To make $S$ total on $[0, d]$, we introduce the additional undefined value $\perp$ that is assigned for every $t \in[0, d]$ where $S(t)$ is undefined. 
This simple definition enables considering basic operators acting on such patterns. For instance, two musical patterns $S_{1}$ and $S_{2}$ with domains $\operatorname{dom}\left(S_{1}\right)=$ $\left[0, d_{1}\right]$ and $\operatorname{dom}\left(S_{2}\right)=\left[0, d_{2}\right]$ can be combined one after the other, in a simple sequential fashion, into a pattern $S_{1} \cdot S_{2}$ with domain $\operatorname{dom}\left(S_{1} \cdot S_{2}\right)=\left[0, d_{1}+d_{2}\right]$ by taking, for all $t \in\left[0, d_{1}+d_{2}\right]$,

$$
\left(S_{1} \cdot S_{2}\right)(t)=\left\{\begin{array}{ll}
S_{1}(t) & \text { when } 0 \leq t<d_{1} \\
S_{2}\left(t-d_{1}\right) & \text { when } d_{1} \leq t \leq d_{1}+d_{2}
\end{array},\right.
$$

where, for convenience, we assume that $S_{1}\left(d_{1}\right)=\perp$.

The simplicity of this basic composition operator makes it appealing for modeling purpose. It can be seen as a timed extension of the well-known concatenation product of strings, and its formal study already led to the rich theory of timed languages [3].

\subsection{Towards an extended model}

It may be argued that this concatenation product is not usable by itself for practical musical applications. In audio processing, for instance, signal continuity is crucial for combining patterns, in order to avoid unwanted artifacts; thus, overlapping patterns to produce smooth cross-fading transitions is highly desirable. In symbolic music modeling, time signatures and associated notions of weak and strong beats (or related notions in non-Western music) may induce constraints on the occurrence dates of musical events. Hence, the arbitrary sequential composition of musical patterns is very likely to lack musical consistency.

Therefore, in both audio and symbolic cases, the sequential composition of patterns generally requires additional parameters that accurately encode how such patterns should be combined one with the other. The basic pattern model introduced so far seems incomplete. It does indicate what musical patterns are but it does not indicate how such patterns can be used.

One may observe that, in music writing, this problem is often solved by the addition of bars. Indeed, in musical scores, musical patterns are not only described as sequences of notes. Bars are added to describe how these musical sequences are to be played (or synchronized) one with the other. As an example, the notion of musical anacrusis refers to a few notes that are to be played before the first logical beat of a musical sequence. Inspired by this synchronization mechanism encoded in music scores, we propose to enhance the aforementioned basic composition of musical patterns in order to allow our model to be practically meaningful. A former study of rhythm structures and rhythm compositions [15] suggests that one may distinguish in every rhythmic pattern a realization window, where the pattern is defined, from a synchronization interval, that describes the usage of that pattern. This model, adapted to musical patterns, is presented here. 


\subsection{Realization and synchronization windows}

In a first approach, the time structure of a musical pattern can be described by the schema illustrated on Figure 1. The musical pattern starts at a given date $s_{1}$ and ends at a given date $s_{4}$. Some subinterval described by two other dates $s_{2}$ and $s_{3}$ indicates, when involved in sequential composition, how this pattern must be combined with others. This situation is depicted on Figure 1.

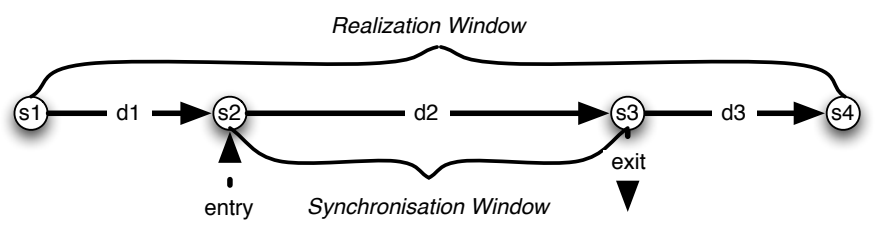

Figure 1: Realization vs synchronisation windows: basic case

Formally, abstracting from the date the pattern is actually fired, the time structure of a pattern $S$ is modeled as a triple of durations

$$
W(S)=\left(d_{1}, d_{2}, d_{3}\right) \in \mathbb{R} \times \mathbb{R}^{+} \times \mathbb{R},
$$

respectively describing durations of what can be called, following the presentation given in [15], the introduction, development and conclusion of pattern $S$.

In this model, as soon as the above dates $s_{1}, s_{2}, s_{3}$ and $s_{4}$ are given, the pattern durations $d_{1}, d_{2}, d_{3}$ are defined such that:

(1) $d_{1}=s_{2}-s_{1}$, with $\left[s_{1}, s_{2}[\right.$ defining the introduction section,

(2) $d_{2}=s_{3}-s_{2}$, with $\left[s_{2}, s_{3}\right]$ defining the development section,

(3) $d_{3}=s_{4}-s_{3}$, with $] s_{3}, s_{4}$ ] defining the conclusion section.

The triple comprising these three specific durations is called time structure of pattern $S$, and denoted by $W(S)$. Time interval $\left[s_{1}, s_{4}\right]$ is the pattern's realization window, and time interval $\left[s_{2}, s_{3}\right]$ is the pattern's synchronization window.

The effective duration $d(S)$ of (the realization window of) a musical pattern $S$ is defined by $d(S)=s_{4}-s 1$ or, equivalently, $d(S)=d_{1}+d_{2}+d_{3}$. It must not be confused with the length of the synchronization window itself, the synchronization duration $s(S)$, defined by $s(S)=d_{2}$.

The model still makes sense when $d_{1}$ and/or $d_{3}$ are negative. For instance, when both $d_{1}$ and $d_{3}$ are negative, introduction and conclusion of $S$ actually correspond to silent sections. In such a case, the induced dates of $S$ are such that $s_{2} \leq s_{1} \leq s_{4} \leq s_{3}$, and the resulting pattern only produces sound from $s_{1}$ to $s_{4}$. The induced time structure is illustrated on Figure 2, with the associated constraint that $d(S)=d_{1}+d_{2}+d_{3} \geq 0$, i.e. the duration of the realization window remains positive. In that case, we have $s(S) \geq d(S)$. 


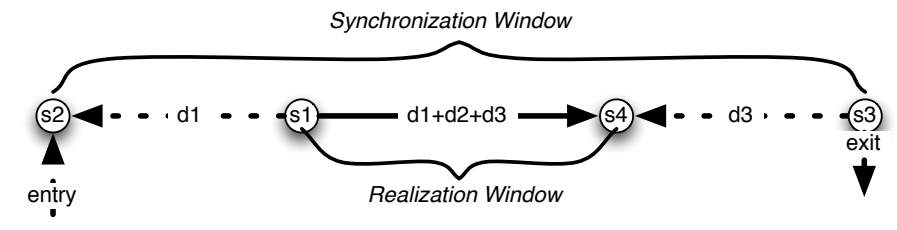

Figure 2: Realization vs synchronisation windows: advanced case

In all cases, the beginning of the synchronization window, at date $s_{2}$, is called entry point of pattern $S$, and the end of the synchronization window, at date $s_{3}$, is called exit point of $S$. Moreover, from now on we define the pattern $S$ with its associated time structure $W(S)=\left(d_{1}, d_{2}, d_{3}\right)$ on the domain

$$
\operatorname{dom}(S)=\left[-d_{1}, d_{2}+d_{3}\right]
$$

where the date 0 always stands for the date of the entry point. Such a definition turns out to be convenient for combining patterns.

When introducing the synchronization window of $S$, we assume that $s(S)=$ $d_{2} \geq 0$. This constraint ensures that the synchronization is defined in a pastto-future manner only. However, one might want to relax such a condition and allow negative synchronization windows. Such a consideration would be helpful from a mathematical point of view: it would result in manipulating a particular monoid structure, namely an inverse monoid [17], in which, by switching the entry and exit points of a given pattern $S$, one could associate the so-called pseudo-inverse of $S$ in inverse monoid theory.

Nevertheless, negative synchronization windows do not yet appear to be relevant for application purpose. In all considered applications, synchronization mechanisms are practically performed from past to future. In the absence of negative synchronization windows, we still obtain concrete instances of monoid structures that have been recently investigated in computer science $[16,14,13]$.

\subsection{Sequential composition}

The purpose of distinguishing synchronization from realization is revealed when defining sequential compositions of musical patterns. This product is defined in two stages as a kind of concatenation of structured patterns. We first show how time structures are combined. Then, from that combination, the effective composition of patterns, possibly with overlaps, is described in detail.

Let $S_{1}$ and $S_{2}$ be two musical patterns with respective time structures $W\left(S_{1}\right)=\left(x_{1}, x_{2}, x_{3}\right)$ and $W\left(S_{2}\right)=\left(y_{1}, y_{2}, y_{3}\right)$. The proposed sequential composition $S_{1} \cdot S_{2}$ of patterns $S_{1}$ and $S_{2}$ consists in placing the synchronization window of $S_{2}$ right after the synchronization window of $S_{1}$. As a result, the two musical patterns are positioned as described on Figure 3. The associated patterns are then assigned the resulting time structure described on Figure 4 with $x=\max \left(x_{1}, y_{2}-x_{2}\right)$ and $y=\max \left(y_{3}, x_{3}-y_{2}\right)$. 


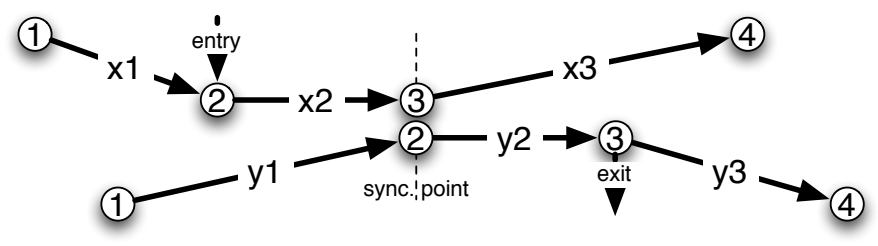

Figure 3: Sequential synchronization of patterns

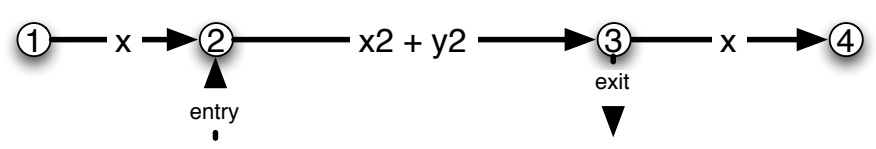

Figure 4: Resulting time structure

Formally, we define $W\left(S_{1} \cdot S_{2}\right)$ by taking

$$
W\left(S_{1} \cdot S_{2}\right)=\left(\max \left(x_{1}, y_{1}-x_{2}\right), x_{2}+y_{2}, \max \left(y_{3}, x_{3}-y_{2}\right)\right)
$$

In this composition, the resulting realization window of the product $S_{1} \cdot S_{2}$ is defined as the union of realization windows of both $S_{1}$ and $S_{2}$. It may be the case that one pattern is completely included into the other; thus, durations of introductory and concluding sections in the resulting time structure are computed by means of a max.

The value of the composition of patterns $S_{1}$ and $S_{2}$ with respective time structures $\left(x_{1}, x_{2}, x_{3}\right)$ and $\left(y_{1}, y_{2}, y_{3}\right)$ is defined as follows: for all $t \in \operatorname{dom}\left(S_{1}\right.$. $\left.S_{2}\right)$,

$$
\left(S_{1} \cdot S_{2}\right)(t)=\left\{\begin{array}{cl}
S_{1}(t) \oplus S_{2}\left(t-x_{2}\right) & \text { when } t \in \operatorname{dom}\left(S_{1}\right) \text { and } \\
& t-x_{2} \in \operatorname{dom}\left(S_{2}\right), \\
S_{1}(t) & \text { when } t \in \operatorname{dom}\left(S_{1}\right) \text { and } \\
& t-x_{2} \notin \operatorname{dom}\left(S_{2}\right), \\
S_{2}\left(t-x_{2}\right) & \text { when } t \notin \operatorname{dom}\left(S_{1}\right) \text { and } \\
& t-x_{2} \in \operatorname{dom}\left(S_{2}\right), \\
\perp & \text { otherwise }
\end{array} .\right.
$$

Note that the sum $\oplus$ of pattern values depends on the type of data to be handled, and the precise semantics of that sum need to be adequately defined depending on the application.

Observe that the hereby defined product is associative w.r.t. the underlying time structures. More precisely, given three musical patterns $S_{1}, S_{2}$ and $S_{3}$, we have $W\left(\left(S_{1} \cdot S_{2}\right) \cdot S_{3}\right)=W\left(S_{1} \cdot\left(S_{2} \cdot S_{3}\right)\right)$. From a mathematical point of view, the resulting algebraic structure is a semigroup. It should be clear that 
the associativity property is expected in order to achieve a computationally robust formalism. Is the resulting product associative on the music patterns themselves? This depends on the associativity of the operator $\oplus$ henceforth on the application field.

In the symbolic case, operator $\oplus$ can be conveniently defined as the union of the sets of events to be performed at a given time. Associativity can thus be guaranteed. In the audio case, operator $\oplus$ is often defined as the average of sample values. In that case, the resulting product is no longer associative. This drawback is however not a surprise as it is commonly encountered in audio signal processing: mixing audio signal is most generally performed by means of some weighted sum of pattern values.

A related logical formalism, based on some interval algebra, was already introduced by Allen [2]. However, in the case of accurate synchronization of music material, interval algebra fails to distinguish temporal issues, e.g. patterns' realization windows may overlap, from logical issues, e.g. patterns' synchronization windows must come one after the other.

Building a logical formalism based on the synchronization algebra presented here may thus refine Allen's logical approach. However, such a logical, hence language theoretical, approach is not presented in this paper. Here, we only provide an algebra for generating musical patterns. The extension of our proposal towards an algebra that generates sets -consequently properties- of patterns, an extension that could then (and only then) be related precisely with Allen's logic, remains to be done.

\subsection{Resynchronization operator}

Given a pattern $S$ with $W(S)=\left(x_{1}, x_{2}, x_{3}\right)$, given $d(S)$ and $s(S)$ the respective realization and synchronization durations of pattern $S$, and given two reals $a$ and $b$ such that $(a-b) d(S) \leq s(S)$, we define the resynchronized pattern $S[a, b]$ by $S[a, b](t)=S(t+a . d)$ for every $t \in \operatorname{dom}(S[a, b])$. This construction, illustrated on Figure 5 where $d$ stands for $d(S)$, is formally defined as follows.

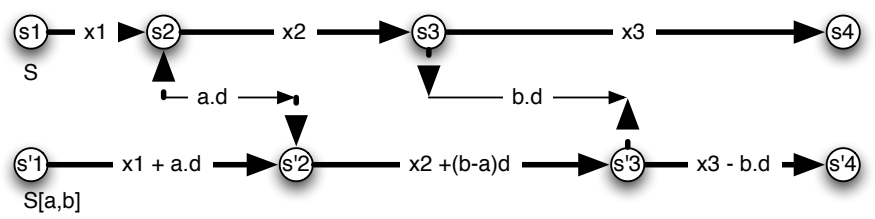

Figure 5: Pattern resynchronization

Keeping 0 as the entry point of $S[a, b]$, we define $\operatorname{dom}(S[a, b])$ from $\operatorname{dom}(S)$ by a translation of -a.d. Reals $a$ and $b$ are respectively called the left offset and the right offset resynchronization ratios of $S$. The underlying time structure is assigned as $W(S[a, b])=\left(x_{1}+a . d(S), x_{2}+(b-a) d(S), x_{3}-b d(S)\right)$. Doing so, we have $d(S[a, b])=d(S)$. 
In other words, the resynchronization operator does not change the realization of the pattern it is applied to, it only modifies its synchronization offsets.

\section{Derived and additional operators}

In this section, we review additional properties of our model. Resynchronization and sequential composition, combined with a new expansion/contraction operator lead to the definition of many practically useful derived operators.

\subsection{Context patterns and left and right shifts}

Patterns $S$ such that $s(S)=0$ are called context patterns. One can check that, provided that $\oplus$ is commutative, then for every context patterns $S$ and $T$ we have $S \cdot T=T \cdot S$, i.e. sequential product on context patterns commute. Observing that the resynchronization pattern $S[a, b]$ of pattern $S$ is a context pattern when $a-b=s(S) / d(S)$, this leads us to define two special context patterns associated to $S$.

The synchronization structure of these new patterns is depicted on Figure 6.

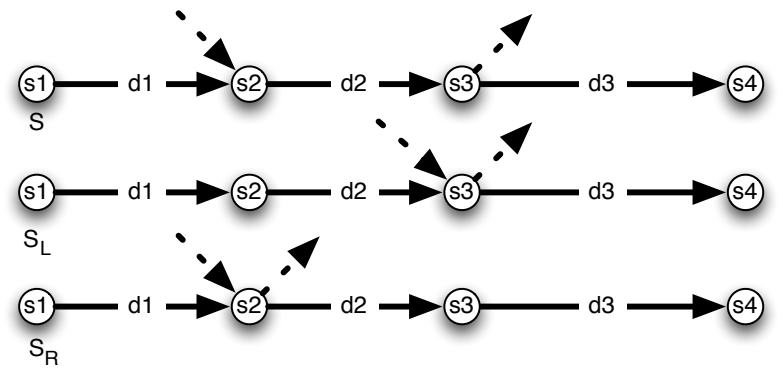

Figure 6: Canonical left and right resynchronizations

When $a=0$ and $b=-s(S) / d(S)$, we call the context pattern $S[a, b]$ the right shift of $S$, and write $S_{R}=S[0,-s(S) / d(S)]$. Practically, $S_{R}$ is obtained from $S$ by shifting its exit point to its entry point, i.e. the sub-pattern in the sync window of $S$ is shifted to the right.

When $a=s(S) / d(S)$ and $b=0$, we call the context pattern $S[a, b]$ the left shift of $S$, and write $S_{L}=S[s(S) / d(S), 0]$. Practically, $S_{L}$ is obtained from $S$ by shifting its entry point to its exit point, i.e. the sub-pattern in the sync window of $S$ is shifted to the left.

If we assume - as aften in the symbolic case - that the sum $\oplus$ involved in the definition of the sequential composition is such that, for all pattern value $v$, $v \oplus v=v$, then the following properties are satisfied:

(1) for all context patterns $S$ and $T, S \cdot S=S$ and $S \cdot T=T \cdot S$, 
(2) for all patterns $S, S \cdot S_{L}=S_{R} \cdot S=S$.

Many more properties are actually satisfied. In fact, the set of patterns equipped with such a sequential composition turns out to be a monoid that is quasi-inverse in some sense [13].

\subsection{Fork and join}

Let $S$ and $T$ be two patterns with respective time structures $W(S)=\left(x_{1}, x_{2}, x_{3}\right)$ and $W(T)=\left(y_{1}, y_{2}, y_{3}\right)$. The fork composition of $S$ and $T$ is defined as the sequential composition $S_{R} \cdot T$. It consists in synchronizing $S$ and $T$ at their entry points, with the resulting synchronization window taken to be that of $T$. This construction is depicted on Figure 7. Similarly, the join composition of $S$

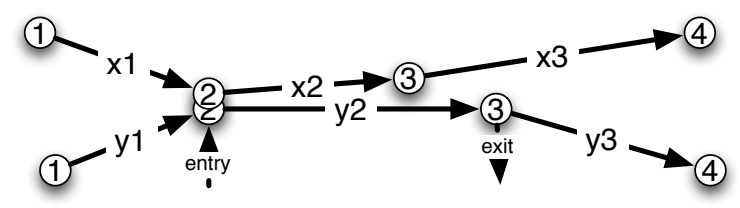

Figure 7: Derived operator: fork

and $T$ is defined as the sequential composition $S \cdot T_{L}$. It consists in synchronizing $S$ and $T$ at their exit points, with the resulting synchronization windows taken to be that of $S$. This construction is depicted on Figure 8 .

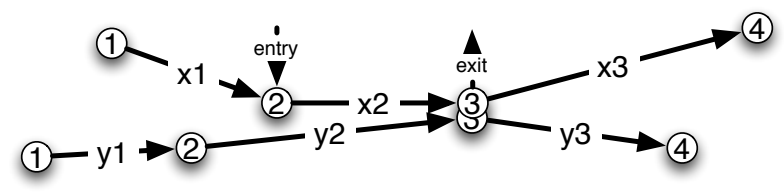

Figure 8: Derived operator: join

Observe that, in general, $S$ and $T$ do not have sync windows of same duration. Since $s\left(S_{R} \cdot T\right)=s(T)$ and $s\left(S \cdot T_{L}\right)=s(S)$, the join and fork operators defined here are not commutative in general.

\subsection{Expansion/contraction operator}

Given a pattern $S$ and a positive real $k$ such that $k \geq 1$ (respectively $k<1$ ), we define the expanded pattern (resp. contracted pattern) $k S$ as the pattern obtained from $S$ by realizing it $k$ times faster (resp. slower). Formally, with $W(S)=\left(x_{1}, x_{2}, x_{3}\right)$, then $W(k S)=\left(k \cdot x_{1}, k \cdot x_{2}, k \cdot x_{3}\right)$ and, for all $t \in \operatorname{dom}(k S)$, $(k S)(t)=S(t / k)$. 
Note that the practical use of this operator may require an additional applicationdependent definition that describes how to modify the realization duration.

By combining resynchronization and expansion/contraction operators on a pattern $S$, we obtain an extended resynchronization operator (or X-resync for short) that modifies the realization of $S$ but preserves the duration of its synchronization window.

More precisely, for every pattern $S$ with $W(S)=\left(x_{1}, x_{2}, x_{3}\right)$, every $a$ and $b$ such that $(a-b) d(S)<s(S)$ (for a non empty resulting sync window), the X-resync operator $S[[a, b]]$ is defined by

$$
S[[a, b]]=\frac{s(S)}{s(S)-(a-b) d(S)} S[a, b] .
$$

By construction, $s(S)=s(S[[a, b]])$. This operator is particularly useful for musical performance applications, as described in Section 5. This operator is illustrated on Figure 9 with $a<0,0<b$, where $v_{1}, v_{2}, \ldots, v_{11}$, stand for pattern values.

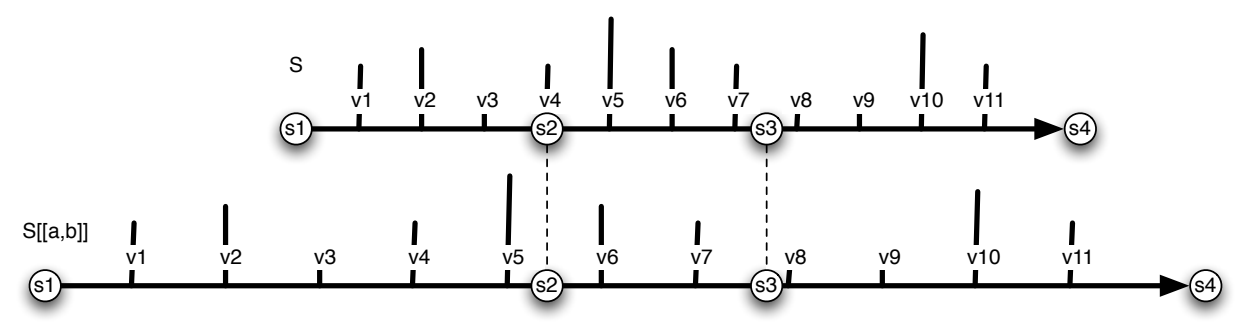

Figure 9: Derived operator: extended resync

\subsection{Parallel and extended parallel products}

When two patterns $S$ and $T$ have sync windows of equal size, i.e. when $s(S)=$ $s(T)$, then not only both $S_{R} \cdot T=T_{R} \cdot S$ and $S \cdot T_{L}=T \cdot S_{L}$, i.e. fork and join arguments commute, but we also have $S_{R} \cdot T=S \cdot T_{L}$, i.e. fork and join coincide. This leads us to define, when $s(S)=s(T)$, the parallel composition of patterns $S$ and $T$ as:

$$
S \| T=S_{R} \cdot T=S \cdot T_{L} .
$$

Combined with expansion/contraction, this product can be generalized. Indeed, provided $s(T) \neq 0$ when $s(S) \neq 0$, we define the asymmetric parallel product $S[[T$ by

$$
S[[T=S \| k T,
$$

with $k=s(S) / s(T)$. In this case, $T$ is expanded or contracted so that its sync window fits the size of the sync window of pattern $S$. In particular, $s(S[[T)=$ $s(S)$. 
By symmetry, provided $s(S) \neq 0$ when $s(T) \neq 0$, we define the asymmetric parallel product $S]] T$ by

$$
S]] T=k S \| T,
$$

with $k=s(T) / s(S)$. In this case, $S$ is expanded or contracted so to fit the synchronization window of $T$. In particular, $s(S]] T)=s(T)$.

These extended parallel products are particularly useful for audio pattern synchronization and reconstruction, as explained in Section 4.

\section{Application to audio patterns: advanced re- composition}

Audio synchronization usually refers to syncing audio patterns to metadata, such as musical scores, lyrics, etc., or to possibly multi-modal syncing, with audio or video material for instance. In this section, we focus on the audioto-audio syncing problem, referred to as audio pattern synchronization. This particular problem has been of major concern over the last decades either for signal processing or music information researchers [21]. Indeed, any system related to the arrangement and organization of several audio patterns must cope with pattern synchronization problems. Applications range from alignment of music data $[22,8]$ to automatic mixing of audio playlists [11], for instance.

This section investigates the use of advanced synchronization in the case of audio pattern handling. Although most of previous works on audio synchronization focus on the accurate syncing of two audio patterns, the formal approach introduced in this paper enables the synchronization of any number of patterns using the aforementioned advanced operators. Moreover, the distinction between synchronization and realization windows turns out to have major practical benefits.

\subsection{Audio advanced synchronization}

Synchronizing audio patterns brings several specific issues that need to be addressed in order to enable a practical use. First, re-synchronization employed with contraction/expansion operators may change the duration of audio patterns. In such cases, most audio applications require expanding or contracting sounds while keeping the original pitch content. Thus, contrastingly to symbolic applications (Section 5), a particular time-stretching function has to be defined. Classical examples of such functions include time-domain signal processing such as Pitch Synchronous Overlap and Add (PSOLA) [20] inspired methods, or frequency domain processing such as advanced phase vocoder techniques (see [9] and references therein).

Another specificity of audio patterns lies in their digital representation, that restricts their codomain to some quantized, finite subset. Let $A$ be this

codomain. Due to the digital audio representation, the sum of digital audio 
patterns must be defined as follows: $\oplus_{A}: A \times A \rightarrow A$. Moreover, when considering audio patterns, the sum of any number of signals must be continuous in order to avoid any audio artifact. An adequate summing function respecting these two criteria may be obtained by multiplying patterns by amplitude masks, and computing the mean of resulting signals. Formally, for an audio pattern $S_{1}$ with time structure $W\left(S_{1}\right)=\left(x_{1}, x_{2}, x_{3}\right)$ and for all $t \in \operatorname{dom}\left(S_{1}\right)$, we define the masked pattern $M\left(S_{1}\right): \operatorname{dom}\left(S_{1}\right) \rightarrow A$ with the same time structure as follows:

$$
M\left(S_{1}\right)(t)=\left\{\begin{array}{ll}
\frac{t+x_{1}}{x_{1}} S_{1}(t) & \text { when }-x_{1} \leq t<0 \\
S_{1}(t) & \text { when } 0 \leq t \leq x_{2} \\
\frac{x_{2}+x_{3}-t}{x_{2}+x_{3}} S_{1}(t) & \text { when } x_{2}<t \leq x_{2}+x_{3}
\end{array} .\right.
$$

We also define the inverse mask pattern $\bar{M}\left(S_{1}\right): \operatorname{dom}\left(S_{1}\right) \rightarrow A$ as the dual of $M\left(S_{1}\right): \bar{M}\left(S_{1}\right)=S_{1}-M\left(S_{1}\right)$.

The sum of two audio patterns is then defined as the arithmetic mean of masked patterns, i.e. for all $t \in \operatorname{dom}\left(S_{1}\right) \cap \operatorname{dom}\left(S_{2}\right)$,

$$
S_{1}(t) \oplus_{A} S_{2}(t)=\frac{1}{2}\left(M\left(S_{1}\right)(t)+M\left(S_{2}\right)(t)\right) .
$$

More generally, the sum of $k \in \mathbb{N}$ overlapping audio patterns is defined, for all $t \in \operatorname{dom}\left(S_{1}\right) \cap \operatorname{dom}\left(S_{2}\right) \cap \cdots \cap \operatorname{dom}\left(S_{k}\right)$, as

$$
\bigoplus_{i=1}^{k} S_{i}(t)=\frac{1}{k} \sum_{i=1}^{k} M\left(S_{i}\right)(t) .
$$

It is worth noting that with such a definition, which ensures that signal continuity is respected, sequentially composing audio patterns may no longer be associative. In sequences where at most two patterns overlap, associativity is respected. However, if three or more patterns overlap each other at some point in their combination, the order in which they are summed may change the resulting pattern. This non-associative property makes real-time audio handling challenging with the present model, in which situation patterns can be expected to be combined in an iterative manner. In such a case, another definition should be considered. For offline applications, on the other hand, this associative property can be relaxed: the overall synchronization structure is established before composing patterns, and the composition may then be realized in an arbitrary order.

\subsection{Application to audio recomposition}

As a practical use of the synchronization theory, we propose to extend the automatic audio assignment method proposed in [19]. The solution proposed in 
that paper is to select one or several parts in an audio recording to reconstruct a missing section. The detection algorithm is based on string alignment methods [7] in order to accurately detect musical repetitions. Although the algorithm is successful in identifying relevant parts, the accurate, possibly iterative, synchronization of reconstructed patterns was left as a perspective. This section is dedicated to using our synchronization model to explicit these advanced signal reconstruction operations.

Let $S$ be an audio piece, and $P$ its missing part. As explained in [19], the section that best fits $P$ can be infered by analysing local information around $P$. The pattern $P^{\prime}$, composed as a development section containing $P$ surrounded by an introduction and a conclusion, has to be synchronized with another pattern $R$ within $S$ that plays the role of replacement pattern. The durations of introductory and concluding parts of $P^{\prime}$ as well as the replacement pattern $R$ are determined by the alignment algorithm [19]. With a properly defined synchronization between $P^{\prime}$ and $R$, one should be able to switch from $P^{\prime}$ to $R$ in order to fill-in the missing part $P$.

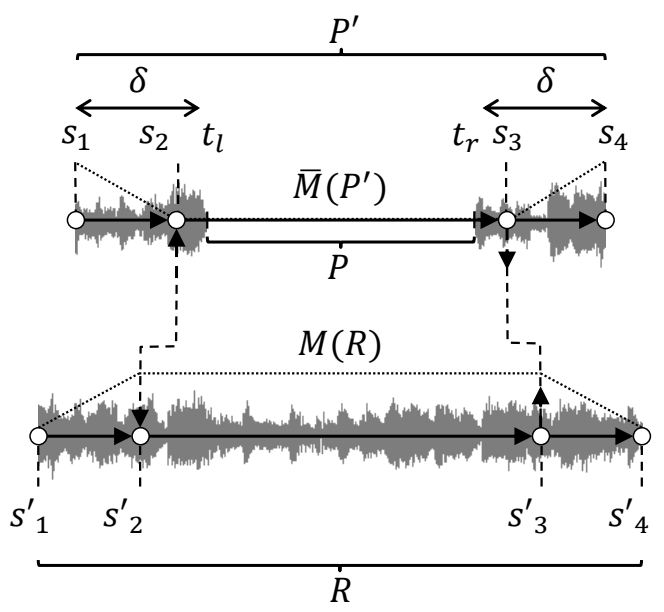

Figure 10: Synchronization structure for re-assigning a missing part $\mathcal{P}$ with one pattern. Dashed lines correspond to synchronization points, while dotted lines show audio masks applied to the composition.

Formally, let $\delta$ be the duration of local search contexts around $P$ (see [19]). We denote by $t_{l}$ the beginning time of $P$ in $S$, and $t_{r}$ the ending time of $P$ in $S$. The beginning date of $P^{\prime}$ is denoted by $s_{1}=t_{l}-\delta$. Similarly, the ending date of $P^{\prime}$ is denoted by $s_{4}=t_{r}+\delta$. As explained above, the alignment algorithm identifies the synchronization points between $P^{\prime}$ and $R$, denoted by $s_{2} \in\left[s_{1}, t_{l}\right]$ and $s_{3} \in\left[t_{r}, s_{4}\right]$ in $P^{\prime}$ and by $s_{2}^{\prime}$ and $s_{4}^{\prime}$ in $R$. Finally, the introduction and conclusion of pattern $R$ are attributed the same durations as those of pattern $P^{\prime}$, hence the realization times of $R$ are defined as $s_{1}^{\prime}=s_{2}^{\prime}-\left(s_{2}-s_{1}\right)$ and $s_{4}^{\prime}=s_{3}^{\prime}+\left(s_{4}-s_{3}\right)$. 
Figure 10 gives an overview of the synchronization structure involved. Synchronization and realization times are provided for both identified patterns $P^{\prime}$ and $R$. Masks are represented as dotted lines.

The overlapping introductions and conclusions in $P^{\prime}$ and $R$ are intended to enable a seamless transition. Therefore, the parallel composition of these patterns must be realized between the inverse mask of $P^{\prime}$ (fading-out, then in), and the mask of $R$ (fading-in, then out). Finally, the reconstructed pattern $r(P)$ is defined as follows:

$$
r(P)=\bar{M}\left(P^{\prime}\right)[[M(R) .
$$

The synchronization operators defined in this paper enables us to bring the reconstruction model one step further. As suggested in [19], a major improvement of the reconstruction method would consist in combining a set of parts that locally fit the the missing data section $P$, especially when $P$ is large. This problem can easily be addressed as a sequential composition of reconstruction sections. Let $k \in \mathbb{N}$ be the number of locally similar parts analysed by an alignment method, and denoted by $R_{1}, R_{2} \cdots R_{k}$. The reconstruction consists in sequentially combining the $k$ distinct parts (adequately masked), and synchronizing the resulting pattern to $P^{\prime}$.

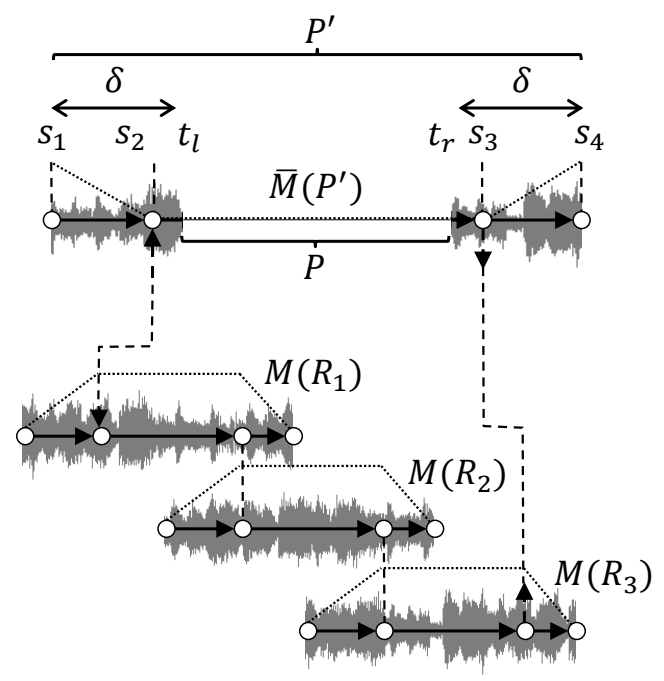

Figure 11: Synchronization structure for re-assigning a missing part $\mathcal{P}$ with 3 patterns employed for reconstruction. Dashed lines correspond to synchronization points, while dotted lines show audio masks applied to the composition.

Figure 11 depicts the applied synchronization for $k=3$. The reconstructed pattern $r_{k}(P)$ is hence defined as follows:

$$
r_{k}(P)=\bar{M}\left(P^{\prime}\right)\left[\left[\left(M\left(R_{1}\right) \cdot M\left(R_{2}\right) \cdot \ldots \cdot M\left(R_{k}\right)\right) .\right.\right.
$$




\section{Application to musical performance: Advanced Live-Looping}

Live-looping is a musical technique that consists in recording loops of data coming from a live musical input. These loops are then synchronized with a pulse, whose period often corresponds to the length of one of the loops.

When looped data is symbolic, consisting of notes for instance, we talk about control live-looping. Various live-looping interfaces exist, such as effects pedals, racks and software applications. New musical instruments such as Fijuu [23] or Drile [4] also rely on this technique and even improve it. For instance, in Drile, this technique is expanded with the creation of live-looping trees, which provide new possibilities in terms of musical structures and loops manipulations.

\subsection{Model of control live-looping}

Live-looping can easily be conceptualized using the model and operators defined in previous sections. For this application, patterns are describing symbolic musical pieces. Each element of the codomain $A$ of a pattern $S$ thus describes a set of control events that have to be triggered simultaneously. The sum $\oplus$ of two elements of $A$ is then defined as the union of sets.

Modeling control live-looping amounts to defining, from each pattern $S$, the pattern $\operatorname{loop}(S)$ that models the infinite sequential product of $S$ with itself. In our formalism, this can be done as follows.

Given such a pattern $S$ with non empty sync window, i.e. $s(S)>0$, we first define the pattern $D(S)$ from pattern $S$ by delaying $S$ by the size $s(S)$ of its sync window. Formally:

$$
D(S)=S[-s(S) / d(S),-s(S) / d(S)] .
$$

Figuring (sets of simultaneous) events by vertical bars, such a self-delay operator is illustrated on Figure 12. By iterating this construction, we define, for every

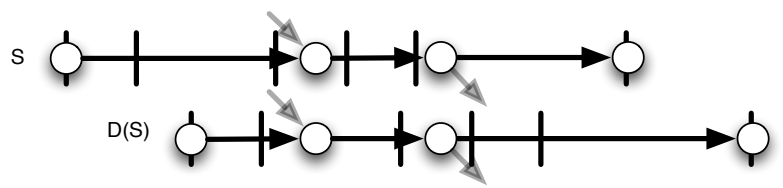

Figure 12: Derived operator: self delay

$k, D^{k+1}(S)=D\left(D^{k}(S)\right)=S[-k . s(S) / d(S),-k . s(S) / d(S)]$. Since all delayed patterns have sync windows of same length, the parallel composition defined in Section 3.4 can be applied.

Formally, we then define the pattern $\operatorname{loop}(S)$ resulting from a looping execution of pattern $S$ as the infinite parallel composition:

$$
\operatorname{loop}(S)=S\|D(S)\| D^{2}(S)\|\cdots\| D^{k}(S)\left\|D^{k+1}(S)\right\| \cdots
$$


This construction is illustrated on Figure 13. Note that in practice, since

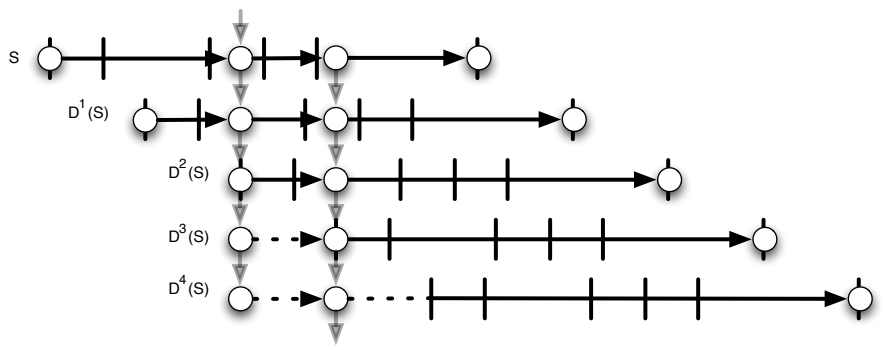

Figure 13: Derived operator: loop

$d(S)$ is finite and $s(S)>0,(\operatorname{loop}(S))(t)$ is finitely defined since, at a given date $t$, only finitely many delayed copy of $S$ are defined, i.e. $D^{k}(S)(t) \neq \perp$ for finitely many $k$.

\subsection{Recalage: an advanced control live-looper}

Combining the above loop operator with the extended resync operator defined in Section 3.3, we eventually define a versatile technique for musical performance which gives a way to generate new musical patterns from previously recorded ones. We implement these ideas in a instrument called Recalage.

Recalage allows one to record and play loops of midi events. When recording a loop, it is automatically synchronized with a multiple of the first previously recorded loop currently playing. Recalage provides controls for triggering loops, i.e. recording them if they are empty, toggling them otherwise, as well as for erasing them. Recalage, as depicted on Figure 14, is currently used with two loops, each represented by a specific widget, although stacking more loops only amounts to adding more loop widgets. For each loop, the current instance of the pattern is drawn in the middle of the widget. The timeline is horizontal; thus, previous and next instances of a loop, which may overlap with the current one, are displayed respectively above and below the current instance. The beginning and ending cues of the realization window are represented by white circles which can be moved in real-time to control the extended resynchonization of the pattern. A white rectangle is added to the middle of the realization window, allowing users to simultaneously modify the begin and end offsets without changing the length of the realization window. For the current instance, the offset control elements (white circles and square) are larger and thicker and can be moved by clicking and dragging them. Recorded events are drawn as black lines. The beginning and end of the synchronization windows, for all instances, are represented by white vertical lines. The part with a lighter background emphasizes the fixed synchronization window of the current instance, played in loop. Finally, a vertical cursor provides a visual feedback on the playback of the loop. In the example depicted on Figure 14, one loop (top) is expanded and 
playing and one (bottom) is contracted and stopped. Observe that at any time, one only need to visualize which events occur in a single sync window. All the available controls on the graphical user interface can also receive MIDI Control Change and MIDI Note messages, so that all the looping and resynchronization operations can be done with an external hardware controller.

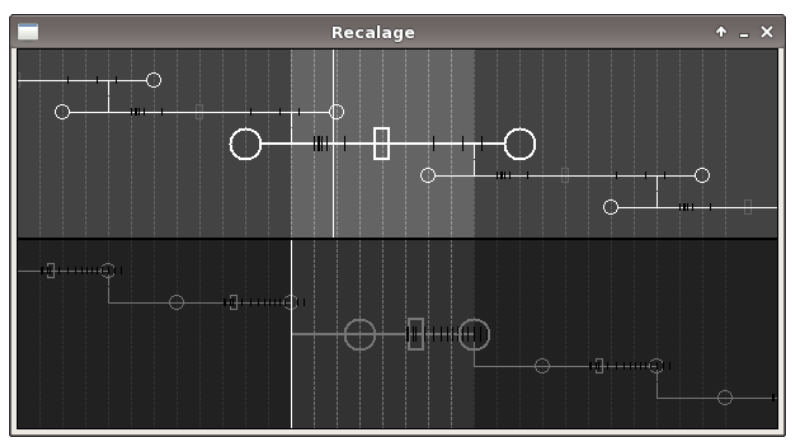

Figure 14: Recalage looper with two loops

Thanks to Recalage, one may notably create complex rhythmic structures out of much simpler ones by applying graphically straightforward transformations. Figure 15 depicts typical musical examples created using Recalage from a simple 4 notes pattern.
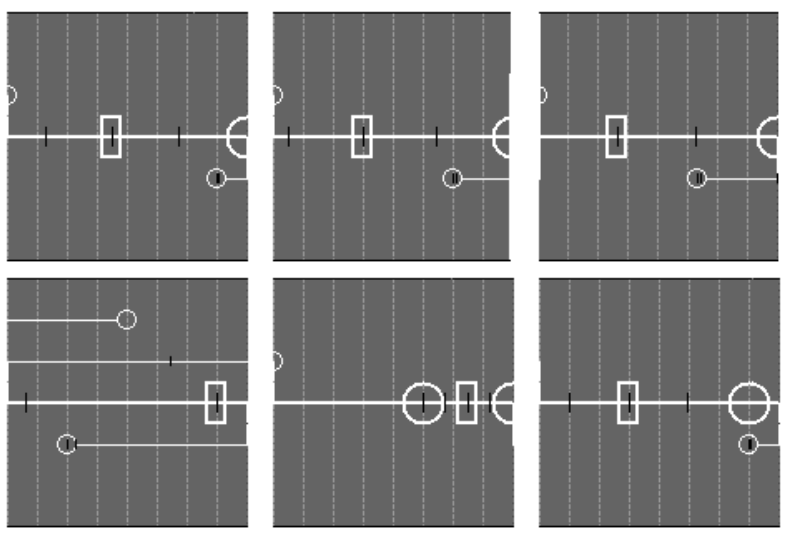

Figure 15: Example manipulations of a $4 / 4$ pattern with Recalage

These examples of musical sequences are created from manipulating a simple $4 / 4$ pattern $S$ made of four quarter notes. From left to right, they can be described as follows.

On the first line, the left expansion $S[[-1 / 8,0]]$ creates a slight shift in the basic $4 / 4$ rhythm that is typically found as anticipation pattern in electronic 
music. The left expansion $S[[-1 / 4,0]]$ echoes a typical salsa bass rhythm : the Tumbao. The left expansion $S[[-1 / 3,0]]$ creates a triplet pattern with the formerly first and fourth beats played together. On the second line, $S[[-3 / 4,1 / 2]]$ sounds like a rather unbalanced pulse. The left contraction $S[[5 / 8,0]]$ emphasize the expectation of the next strong beat as all played notes are pushed to the right. Finally, the left shift $S[[-1 / 8,-1 / 8]]$ moves the whole pattern to the left by half a beat. Instead of having to erase his rhythmic loop and record a new one, a musician using Recalage may therefore easily change rhythmic signature for example between two parts of a melodic improvisation. For every derived looping pattern, the underlying pulse remains the same since the length of sync windows remains equal to the original. Therefore the superimposition of various patterns allows for the creation of many musical contrasts. For instance, the $4 / 4$ pattern $S$ played with the triplet pattern $S[[-1 / 3,0]]$ creates a typical 3 on 4 poly-rhythm.

\section{Conclusion}

In this paper, we propose a rich algebra for manipulating and synchronizing musical patterns. From a generic model for representing musical events, we distinguish the notions of realization and synchronization of a pattern. We then define several meaningful advanced synchronization operators and emphasize their practical relevance. A case study for a concrete audio application is presented, in which the model is employed to explicit the re-assignment of missing audio parts. The practical use of our model is also presented in a particular application of symbolic pattern manipulation, namely control live-looping. The introduced live-looper Recalage allows musicians to obtain rich rhythmic structures out of re-synchronizing simpler ones, and to combine them in a versatile graphical interface. For both symbolic and audio applications, our model is successful in formally describing and expliciting complex synchronization problems.

\section{References}

[1] C. Agon, J. Bresson, and G.Assayag. The OM composer's Book, Vol.1 $\mathcal{E}$ Vol.2. Collection Musique/Sciences. Ircam/Delatour, 2006.

[2] J.F. Allen. Maintaining knowledge about temporal intervals. Communications of the ACM, 26(11):832-843, 1983.

[3] R. Alur and D. L. Dill. A theory of timed automata. Theor. Comput. Sci., 126(2):183-235, 1994.

[4] F. Berthaut, M. Desainte-Catherine, and M. Hachet. Drile : an immersive environment for hierarchical live-looping. In Proceedings of New Interfaces for Musical Expression (NIME10), pages 192-197, Sydney, Australia, 2010. 
[5] A. Cipriani and M. Giri. Electronic Music and Sound Design - Theory and Practice with Max/Msp. Contemponet, 2010.

[6] A. Cont. Antescofo: Anticipatory synchronization and control of interactive parameters in computer music. In International Computer Music Conference (ICMC), 2008.

[7] M. Crochemore, C. Hancart, and T. Lecroq. Algorithms on strings. Cambridge University Press, 2007.

[8] S. Dixon and G. Widmer. Match: A music alignment tool chest. Proc. of the 6th International Society for Music Information Retrieval Conference (ISMIR), 2005.

[9] A.J.S. Ferreira. An odd-dft based approach to time-scale expansion of audio signals. Speech and Audio Processing, IEEE Transactions on, 7(4):441-453, 1999.

[10] D. Fober, Y. Orlarey, and S. Letz. Faust architectures design and osc support. In 14th Int. Conference on Digital Audio Effects (DAFx-11), pages 231-216. IRCAM, 2011.

[11] H. Ishizaki, K. Hoashi, and Y. Takishima. Full-automatic dj mixing system with optimal tempo adjustment based on measurement function of user discomfort. In Proc. of ISMIR, 2009.

[12] D. Janin. A lazy real-time system architecture for interactive music. In Actes des Journées d'Informatique Musicales (JIM 2012), pages 133-139, Mons, Belgique, 2012.

[13] D. Janin. Quasi-inverse monoids. Technical Report RR-1459-12, LaBRI, Université de Bordeaux, February 2012.

[14] D. Janin. Quasi-recognizable vs MSO definable languages of onedimensional overlaping tiles. In Mathematical Foundations of computer Science (MFCS), volume 7464 of LNCS, pages 516-528, 2012.

[15] D. Janin. Vers une modélisation combinatoire des structures rythmiques simples de la musique. Revue francophone d'informatique musicale (RFIM), 2,2012 .

[16] D. Janin. On languages of one-dimensional overlapping tiles. In International Conference on Current Trends in Theory and Practice of Computer Science (SOFSEM), volume (to appear) of LNCS, 2013.

[17] M. V. Lawson. Inverse Semigroups : The theory of partial symmetries. World Scientific, 1998.

[18] S. Letz, Y. Orlarey, and D. Fober. Real-time composition in Elody. In Proceedings of the International Computer Music Conference, pages 336339. ICMA, 2000. 
[19] B. Martin, P. Hanna, V. Ta, M. Desainte-Catherine, and P. Ferraro. Exemplar-based assignment of large missing audio parts using string matching on tonal features. In Proc. of ISMIR, pages 507-512, 2011.

[20] E. Moulines and F. Charpentier. Pitch-synchronous waveform processing techniques for text-to-speech synthesis using diphones. Speech communication, 9(5):453-467, 1990.

[21] M. Müller. New developments in music information retrieval. In Proc. 42nd AES Conf, pages 11-20, 2011.

[22] M. Müller, H. Mattes, and F. Kurth. An efficient multiscale approach to audio synchronization. Proc. of ISMIR, pages 192-197, 2006.

[23] J. Oliver and S. Pickles. Fijuu2: a game-based audio-visual performance and composition engine. In NIME '07: Proceedings of the 7th international conference on New interfaces for musical expression, pages 430-430, New York, NY, USA, 2007. ACM. 